\title{
Contrast enhanced electron beam computed tomography to analyse the coronary arteries in patients after acute myocardial infarction
}

\author{
S Achenbach, D Ropers, M Regenfus, G Muschiol, W G Daniel, W Moshage
}

\begin{abstract}
Objective-To evaluate the accuracy of contrast enhanced electron beam computed tomography (EBCT) after acute myocardial infarction in determining patency of the infarct related artery and detecting high grade stenoses and occlusions in the coronary vessels.

Design-Case study using blinded comparison with invasive coronary angiography.

Patients-36 patients (mean age 53 years) 4-70 days after acute myocardial infarction.

Interventions-The patients were studied by EBCT and invasive coronary angiography. For EBCT, 50 axial images of the heart ( $3 \mathrm{~mm}$ slice thickness) were acquired. They were triggered by the ECG during breath holding, after intravenous injection of contrast agent. The original images, surface reconstructions, and maximum intensity projections were evaluated for the presence of high grade stenoses and occlusions of the coronary arteries.
\end{abstract}

Main outcome measures-EBCT results were compared with invasive coronary angiography. Results-Of a total of 144 coronary arteries (left main, left anterior descending, left circumflex, and right coronary artery in 36 patients), 29 (20\%) were unevaluable by EBCT. In the remaining arteries, 33 of 36 high grade lesions were correctly detected ( $92 \%$ sensitivity). Specificity was also $92 \%(73 / 79)$. Patency of the infarct related artery was correctly detected in 15 of 16 cases (94\%). Five of the 14 occluded infarct related arteries (35\%) were mistaken as stenotic but patent, and six could not be assessed.

Conclusions-EBCT is very accurate in detecting significant coronary artery lesions in patients after acute myocardial infarction, but differentiation between occluded and patent infarct related arteries is currently unreliable.

(Heart 2000;84:489-493)

Keywords: electron beam CT; coronary angiography; myocardial infarction; computed tomography

In recent years, various non-invasive imaging techniques have shown potential in the visualisation of the coronary arteries and in detecting coronary artery stenoses and occlusions, in particular magnetic resonance imaging ${ }^{1-8}$ However, promising results have also been obtained using electron beam computed tomography (EBCT, "ultrafast CT") ${ }^{9-16}$ However, in most published reports comparing EBCT with invasive coronary angiography, the prevalence of coronary artery disease in the patients studied has been low $\left(57-76 \%^{11}{ }^{13-15}\right)$, and as a result the incidence of high grade coronary artery stenoses (exceeding $75 \%$ diameter reduction) has been very low. In the present study we evaluated the accuracy of EBCT in a group of patients who were referred for invasive angiography after acute myocardial infarction. Our aim was to assess how well the method could determine patency of the infarct related artery, and whether it was capable of detecting high grade coronary artery stenoses and occlusions in a clinically defined group of patients with a high likelihood of significant coronary artery disease.

\section{Methods}

PATIENTS

We studied 36 consecutive patients (29 men, seven women; mean age 53 years) who were scheduled for invasive coronary angiography after acute myocardial infarction. The time interval between the infarct and the EBCT studies was between 4-70 days (mean 17 days). Invasive coronary angiography was performed one day after EBCT. Twenty three of the 36 patients $(64 \%)$ had received thrombolytic treatment, and $33(92 \%)$ had $Q$ wave infarctions. The infarct related artery was the left anterior descending coronary artery in 18 patients, a large diagonal branch in one, the right coronary artery in 13, and the left circumflex coronary artery in four.

Exclusion criteria were: invasive coronary angiography scheduled within less than five days from the time of the infarct; unstable clinical condition; atrial fibrillation; aortocoronary bypass grafts or intracoronary stents; or contraindications to the use of an iodinated contrast agent. However, none of the recruited patients had to be excluded on any of these grounds.

Written informed consent was obtained from all patients. The study protocol was approved by the institutional review committee.

ELECTRON BEAM COMPUTED TOMOGRAPHY EBCT is a cross sectional imaging technique similar to conventional CT. However, the lack of a rotating $x$ ray tube permits a very high temporal resolution: one image can be acquired in $100 \mathrm{~ms}$, which almost completely eliminates image artefacts caused by cardiac motion. In addition, image acquisition can be triggered to the patient's ECG ${ }^{17}{ }^{18}$ 

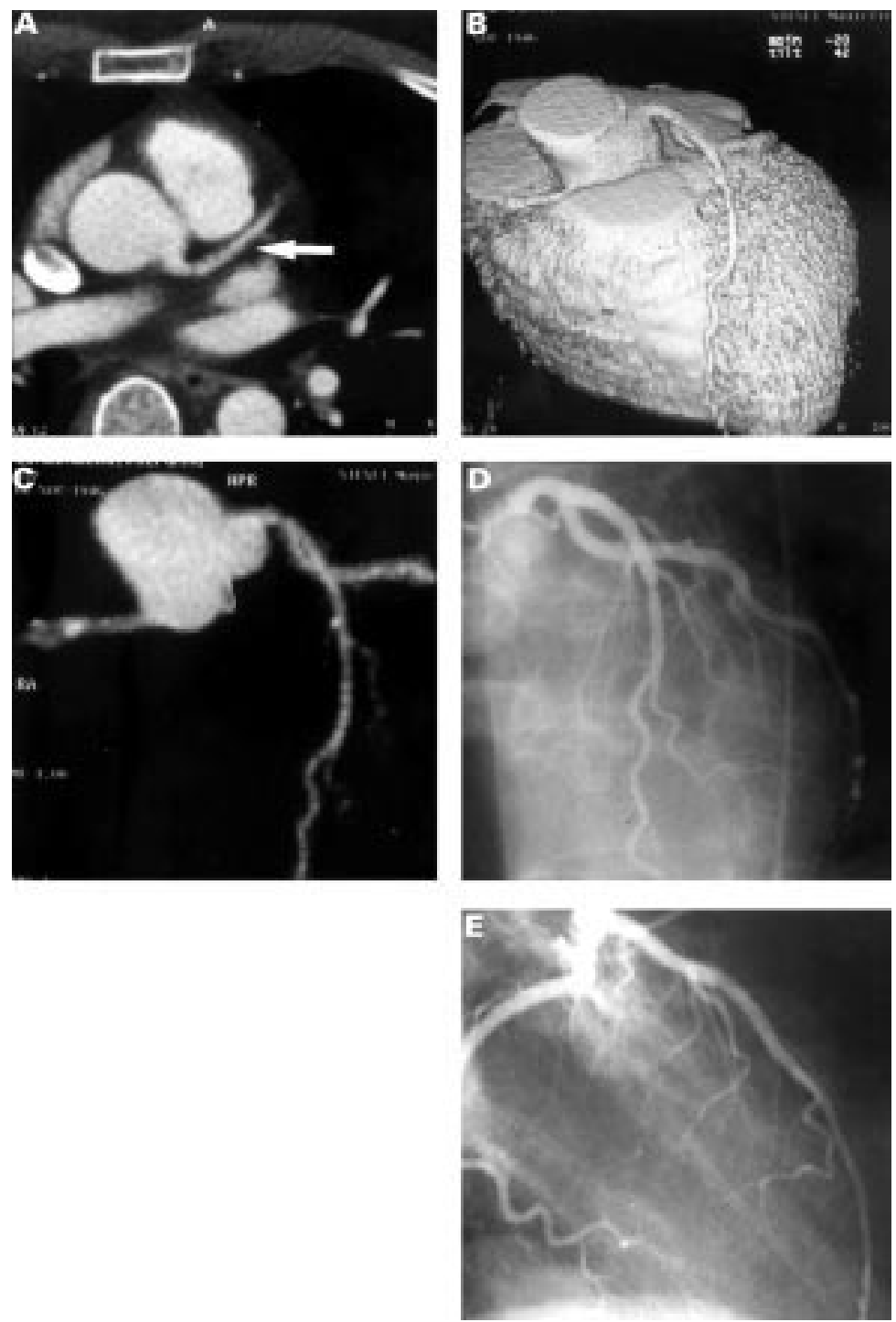

Figure 1 Contrast enhanced EBCT study in a 36 year old male patient after anterior myocardial infarction, without significant stenoses on invasive coronary angiography. $(A)$ Contrast enhanced tomogram at the level of the aortic root shows the left main and left anterior descending coronary artery (arrow). (B) Three dimensional reconstruction of the heart showing left anterior descending coronary artery without significant stenosis. The surface reconstruction is thresholded to show only the contrast enhanced lumen of the coronary arteries. (C) Maximum intensity projection of the coronary arteries showing left anterior descending coronary artery without stenosis. (D, E) Invasive coronary angiography: absence of significant stenoses in the left anterior descending coronary artery after anterior myocardial infarction.

IMAGING PROTOCOL

The investigation was performed using a C-150 XP EBCT scanner (Imatron Inc, South San Francisco, California, USA). Patients were scanned in the supine position. After determination of the heart position and measurement of the contrast agent transit time as described elsewhere, ${ }^{9} 50$ axial cross sections of the heart were acquired during inspiratory breath holding. Triggered to the ECG, one image was acquired after every heart beat. The timing for image acquisition was originally chosen to be $80 \%$ of the RR interval. However, after the 14 th patient, the trigger instant was changed to $40 \%$ of the cardiac cycle for the remaining 22 patients, in order to reduce motion artefacts of the right coronary artery. Slice thickness was $3 \mathrm{~mm}$, with a table feed of $2 \mathrm{~mm}$ between two consecutive acquisitions in order to generate overlapping cross sections. Images were reconstructed with a field of view of $15 \mathrm{~cm}$ and a matrix of $512 \times 512$ pixels to make full use of the scanner's spatial resolution (7 line pairs/ $\mathrm{cm})$. According to the duration of data acquisition - which depended on the patient's heart rate -120 to $160 \mathrm{ml}$ of contrast agent (Ultravist 370 (iopromide); Schering AG, Berlin, Germany) were injected at a flow rate of $4 \mathrm{ml} / \mathrm{s}$ through an $18 \mathrm{G}$ venous access in the antecubital vein. The estimated radiation dose for this imaging protocol is $1.7 \mathrm{mSv} .{ }^{19}$

\section{DATA EVALUATION}

The image sets obtained were transferred to an off-line work station for analysis. After evaluation of the original images, both three dimensional shaded surface display reconstructions and cross sectional maximum intensity projections were generated after manual segmentation of the original images. To generate three dimensional shaded surface display reconstructions, the atrial appendages and pulmonary trunk were removed (fig 1). Shaded surface display reconstructions were then produced using a lower threshold of 80-100 HU to visualise the contrast enhanced vessel lumen selectively, neglecting vessel walls and perivascular connective tissue. ${ }^{9}{ }^{10}$ The surface reconstructions were rendered in various angles to depict all parts of the coronary arteries.

To generate maximum intensity projections - a cross sectional projectional display in which every pixel is assigned a grey scale value corresponding to its actual attenuationthe original datasets were segmented further to remove everything but the coronary arteries. Maximum intensity projections were then rendered in multiple views to depict all parts of the coronary arteries without overlap (fig 1). The original images and both forms of reconstruction were evaluated in conjunction.

A significant lesion was assumed to be present if it was detected in at least one of these forms of reconstruction. Vessel segments which showed extensive calcification in the original images and maximum intensity projections (calcification covering more than half of the artery diameter in at least two consecutive images) were excluded from further analysis, as were vessels with artefacts caused by motion or inconsistent breath holding.

\section{COMPARISON WITH INVASIVE CORONARY}

ANGIOGRAPHY

Two evaluations were performed in every patient. In a first step, all coronary arteries were analysed for the presence or absence of high grade coronary artery stenoses (more than $75 \%$ diameter reduction) or occlusions in the proximal and mid segments of the vessel (segments $1,2,5,6,7$, first half of $8,11,12$, and 13 , according to the American Heart Association classification $\left.^{20}\right)$. Three categories were used for the classification of each artery: presence of high grade stenosis or occlusion, absence of high grade stenosis and occlusion, and unevaluable. The EBCT results were compared with invasive coronary angiography in a blinded fashion; this was done independently 

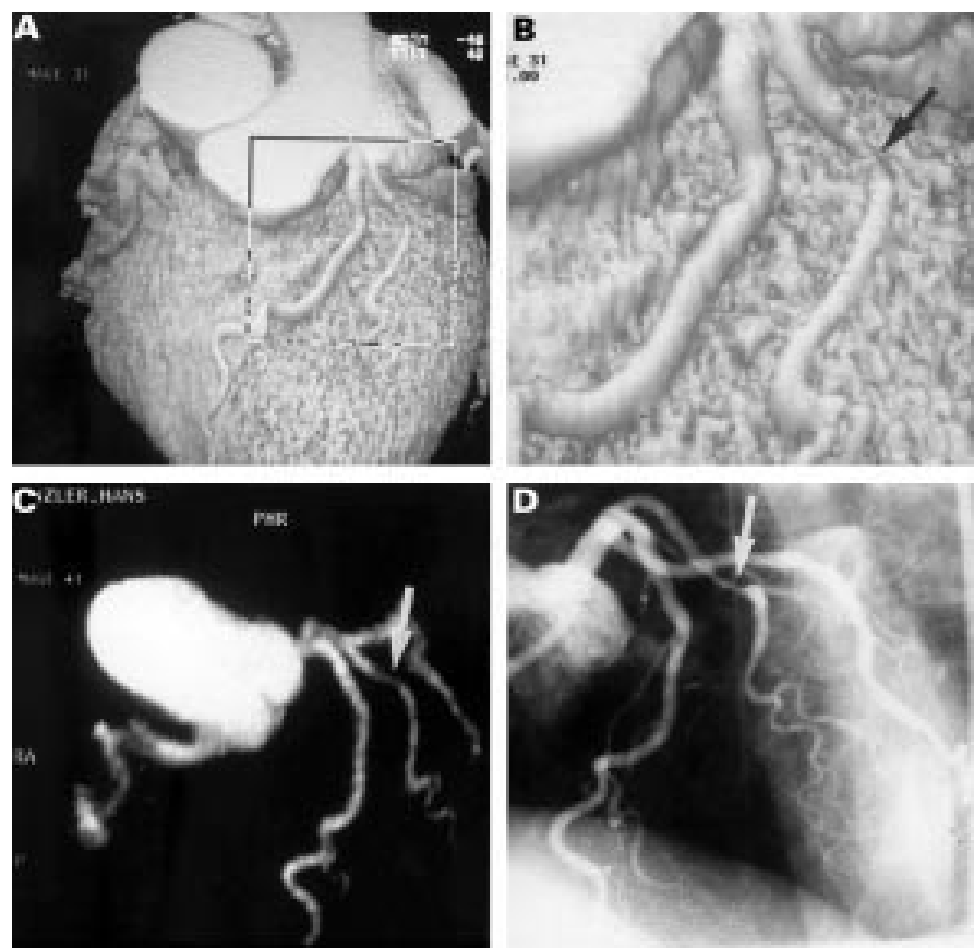

Figure 2 Thirty nine year old male patient after anterolateral myocardial infarction. $(A$, $B)$ Surface reconstruction shows a high grade stenosis in a large diagonal branch (arrow). (C) Maximum intensity projection of the coronary arteries (arrow: stenosis of diagonal branch). (D) Invasive coronary angiography confirms high grade stenosis of the diagonal branch (arrow).
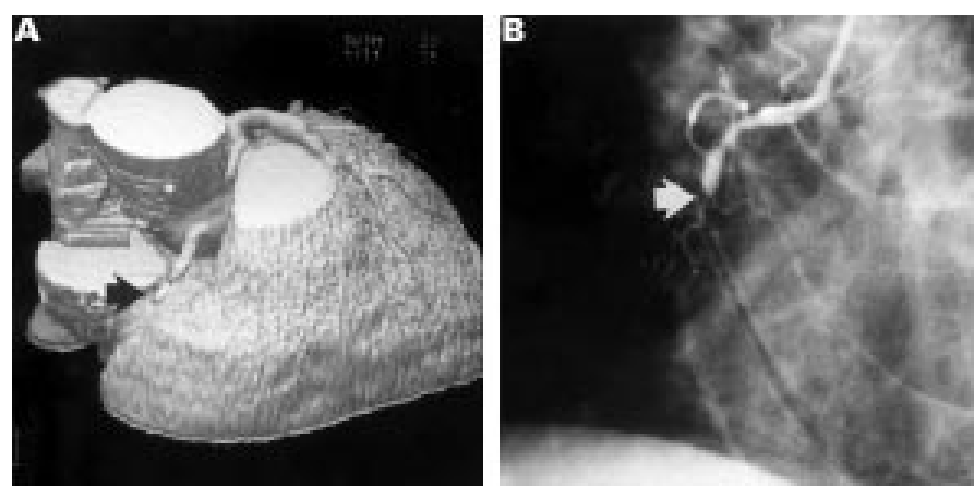

Figure 3 Sixty four year old male patient after posterior myocardial infarction.

(A) Shaded surface display: occlusion of the right coronary artery (arrow). (B) Invasive coronary angiography confirms occlusion of the right coronary artery (arrow).

of the EBCT team by two cardiologists who were unaware of the EBCT results.

In a second evaluation, the infarct related artery (as determined by invasive coronary angiography), but not its angiographic status (presence of stenosis or occlusion), was made known to the EBCT investigators. Based on the original EBCT data and the reconstructed images, an attempt was made to decide whether the infarct related artery was patent or occluded. Three categories were used to classify the infarct related artery: occluded, patent, and unevaluable.

\section{Results}

DETECTION OF HIGH GRADE STENOSES AND OCCLUSIONS

In an initial evaluation, EBCT was used to assess all coronary arteries for the presence of high grade coronary artery stenoses and occlu- sions in the proximal and mid artery segments. Overall, 29 vessels $(20 \%)$ were unevaluable (left main 6; left anterior descending 9; left circumflex 7; right coronary 7). The most common reason for impaired evaluability was extensive calcification of the coronary arteries (19 cases). In the remaining 115 arteries, 33 of 36 high grade stenoses and occlusions were correctly detected by EBCT (92\% sensitivity). Specificity was $92 \%(73 / 79)$, positive predictive value was $85 \%$ (33/39), and negative predictive value was $92 \%(73 / 79)$. Three arteries had either high grade stenoses $(n=2)$ or an occlusion $(\mathrm{n}=1)$ in their distal segments, and no attempt had been made to visualise these by EBCT.

When the axial source images were evaluated alone, the sensitivity for detecting high grade coronary artery stenoses was $86 \%(31 / 36)$ and the specificity $92 \%$ (73/79). For maximum intensity projections, the sensitivity was $89 \%$ $(32 / 36)$ and the specificity was also $89 \%$ (70/79). Shaded surface display reconstructions showed $64 \%$ sensitivity $(23 / 36)$ and $94 \%$ specificity (74/79).

In four patients, EBCT had shown complete absence of significant coronary artery stenoses in the proximal and mid segment of all coronary vessels. In all of these patients, invasive coronary angiography confirmed the absence of significant coronary lesions in these vessel segments (see fig 1). However, in two of the four patients, one lesion was present in a distal coronary artery segment - one high grade stenosis in the distal left circumflex coronary artery, and one occlusion of the distal left anterior descending coronary artery. Neither of these lesions was the target of a revascularisation procedure.

DETERMINATION OF PATENCY AND OCCLUSION OF THE INFARCT RELATED ARTERY

In a second evaluation, the status of the infarct related artery was assessed by EBCT. Thirty of the 36 infarct related arteries (83\%) could be evaluated. Of these, 16 were patent on invasive angiography (left anterior descending 10; diagonal branch 1 ; left circumflex 2; right coronary 3). In EBCT, 15 of these 16 arteries (94\%) were correctly classified as patent (figs 1 and 2). Fourteen of the evaluable infarct related arteries were occluded on invasive coronary angiography (left anterior descending 5; left circumflex 2; right coronary 7). Occlusion was correctly determined in EBCT in nine of these 14 cases (64\%; fig 3). Five occluded arteries were mistaken as patent. In one of these, invasive angiography revealed an occlusion in the distal segment which was not reliably visualised by EBCT; in the other four, filling of the distal vessel segment through collaterals caused the infarct related artery to appear stenotic but patent in the EBCT images (fig 4).

Based on ECG criteria (ST segment normalisation within 120 minutes after initiation of thrombolytic treatment or on admission ${ }^{2122}$ ), patency of the infarct related artery was likely in 16 of 21 patients with patent arteries at angiography (sensitivity $76 \%$ ), and unlikely in 


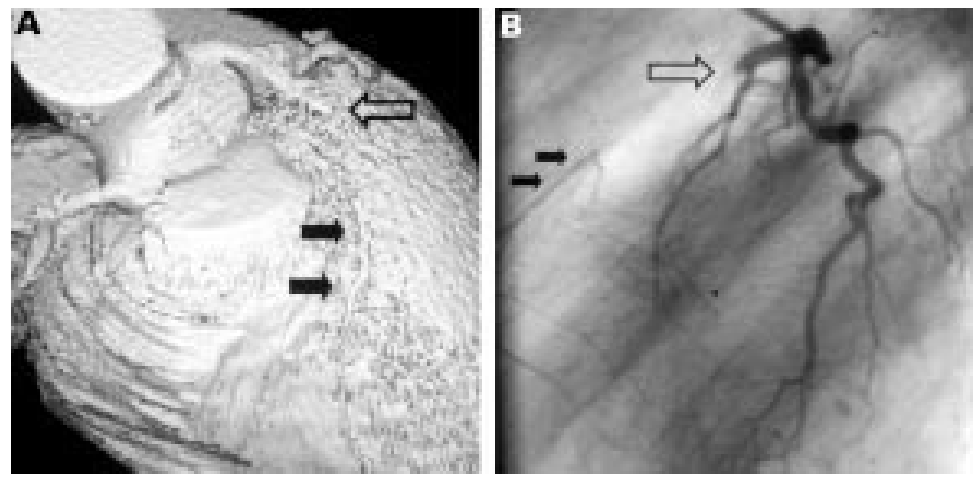

Figure 4 Thirty six year old male patient after anterior myocardial infarction. (A) On EBCT, a long subtotal stenosis of the left anterior descending coronary artery is diagnosed. (B) Invasive coronary angiography shows complete occlusion of the left anterior descending coronary artery (large arrow) with filling of the distal vessel segments via collaterals (small arrows).

Table 1 Comparisons of contrast enhanced EBCT and invasive coronary angiography

\begin{tabular}{llllll}
\hline Author & $n$ & Image evaluation & $\begin{array}{l}\text { Sensitivity } \\
(\%)\end{array}$ & $\begin{array}{l}\text { Specificity } \\
(\%)\end{array}$ & $\begin{array}{l}\text { Unevaluable } \\
(\%)\end{array}$ \\
\hline Nakanishi $^{11}$ & 37 & Tomograms, MPR & $74^{\star}$ & $91^{\star}$ & NA \\
Budoff $^{12}$ & 52 & VRT & 78 & 91 & 11 \\
Reddy $^{13}$ & 23 & Tomograms, MIP & 88 & 79 & 8 \\
Schmermund $^{14}$ & 28 & Tomograms, SSD, MIP & 82 & 88 & 12 \\
Rensing $^{15} \dagger$ & 37 & Tomograms, VRT & 77 & 94 & 19 \\
Achenbach $^{16}$ & 125 & Tomograms, SSD & 92 & 94 & 25 \\
This study & 36 & Tomograms, SSD, MIP & 92 & 92 & 20 \\
\hline
\end{tabular}

${ }^{\star}$ Unevaluable arteries were included in calculation of sensitivity and specificity.

tImaging protocol was based on $1.5 \mathrm{~mm}$ cross sections.

MIP, maximum intensity projection; MPR, multiplanar reconstruction; NA, not applicable; SSD, shaded surface display; VRT, volume rendering technique.

nine of 15 patients with occluded arteries (specificity $60 \%$ ).

\section{Discussion}

In this study of 36 patients after acute myocardial infarction, contrast enhanced EBCT confirmed the accuracy of the technique for detecting high grade coronary artery lesions. However, approximately $20 \%$ of the coronary arteries had to be excluded because of impaired evaluability, which in most cases was caused by extensive calcification. The rate of unevaluable arteries was no higher in this group of patients after myocardial infarction than in previous studies performed at our institution. ${ }^{16}$ Nevertheless, it is evident that improvements in the technology are necessary. While the presence of high grade lesions could be detected with high accuracy by EBCT, the technique did not discriminate between stenoses exceeding $75 \%$ diameter reduction and complete occlusions. Thus it was not possible to differentiate reliably between occlusions and high grade stenoses. As previously demonstrated, the partial volume effect of CT imaging causes very tight stenoses to appear as complete interruptions of the vessel lumen, both in the original tomograms and in the reconstructed images. ${ }^{23}$ High grade stenosis is usually assumed to be present if there is opacification of the vessel lumen distal to the lesion, and so occlusions can be misclassified as stenoses if the distal vessel segments are filled through collaterals. ${ }^{23}$ For this reason, the imaging protocol used in this study-while detecting patency with high sensitivity - did not reliably identify occlusions of the infarct related artery. While EBCT performed better than ECG criteria $^{21} 22$ in detecting patent infarct related arteries, occluded arteries were detected more reliably on ECG. In the same way, complete or incomplete reperfusion (TIMI $3 v$ TIMI 0,1 , or 2 flow in angiography) could not be determined by EBCT. This limits the potential clinical application of the method in patients after thrombolytic treatment of acute myocardial infarction or in unstable coronary syndromes. Different imaging protocols which investigate flow dynamics in the coronary $\operatorname{arteries}^{2425}$ and higher spatial resolution, which can be achieved with a newly available detector system for the EBCT scanner and should reduce partial volume effects, may help to overcome this problem.

The patients in this investigation had an increased prevalence of high grade coronary artery stenoses compared with those in previously published reports. ${ }^{11-16}$ The sensitivity and specificity for detecting high grade coronary artery lesions in our study were higher than reported by other groups (table 1). ${ }^{11-15}$ The reason for these differences may be that previous investigators assessed the accuracy of EBCT for detecting stenoses exceeding 50\% diameter reduction, while we attempted identification of stenoses exceeding $75 \%$ reduction. In addition, we excluded a larger number of arteries from evaluation because of impaired image quality. Some investigators also used different image acquisition protocols ${ }^{15}$ or different forms of image reconstruction. ${ }^{11}{ }^{12}$ The negative predictive value of $92 \%$ in our study is in accordance with the high negative predictive value found in all previous studies.

Maximum intensity projections showed higher accuracy than shaded surface display reconstructions for the detection of high grade coronary artery stenoses and occlusions, while the evaluation of the original source images permitted almost the same accuracy. By combining all forms of image display, there was a further slight increase in accuracy. Newer forms of image reconstruction - such as the volume rendering technique-may be even better suited to visualising the coronary arteries, especially as these techniques may enable calcification inside the coronary artery lumen to be demonstrated.

In the course of our study, we changed the trigger on the image acquisition protocol from $80 \%$ of the RR interval to $40 \%$. By doing this, we obtained a pronounced reduction in movement artefacts, especially in the right coronary artery. However, the numbers were too small for statistical analysis (14 v 22 patients). The optimal timing of image acquisition within the cardiac cycle should be investigated further to improve the results of this technique.

\section{CONCLUSIONS}

Contrast enhanced EBCT is very accurate for detecting high grade coronary artery stenoses, especially in patients who have had myocardial infarcts, in whom there will be a high prevalence of significant coronary lesions. Limitations of this otherwise promising technique are that occlusions and stenoses of the 
coronary arteries cannot be reliably differentiated, and that a substantial number of coronary vessels cannot be evaluated.

1 Manning WJ, Li W, Edelman RR. A preliminary report comparing magnetic resonance coronary angiography with conventional angiography. $\mathrm{N} \mathrm{Engl} \mathcal{F}$ Med 1993;328:828-32.

2 Post JC, van Rossum AC, Hofman MB, et al. Threedimensional respiratory-gated MR angiography of coronary arteries: comparison with conventional coronary angiography. Am f Roentgenol 1996;166:1399-404.

3 Pennell DJ, Bogren HG, Keegan J, et al. Assessment of coronary artery stenosis by magnetic resonance imaging. Onary artery stenosis

4 Müller MF, Fleisch M, Kroecker R, et al. Proximal coronary artery stenosis: three-dimensional MRI with fat saturation and navigator echo. F Magn Reson Imaging 1997;7:644-51.

5 Kessler W, Achenbach S, Moshage W, et al. Usefulness of respiratory gated magnetic resonance coronary angiography in assessing narrowings $>$ or $=50 \%$ in diameter in native coronary arteries and in aortocoronary bypass grafts. Am f Cardiol 1997;80:989-93.

6 Wielopolski PA, van Geuns RJM, de Feyter PJ, et al. Coronary arteries. Eur Radiol 1998;8:873-85.

7 Goldfarb JW, Edelman RR. Coronary arteries: breath-hold, gadolinium-enhanced, three-dimensional MR angiography. Radiology 1998;206:830-4.

8 Sandstede JJW, Pabst T, Beer M, et al. Three-dimensional MR coronary angiography using the navigator technique compared with conventional coronary angiography. $A m \mathcal{F}$ Radiol 1999;172:135-9.

9 Moshage W, Achenbach S, Seese B, et al. Coronary artery stenoses: three-dimensional imaging with electrocardiographically triggered, contrast agent-enhanced, electrongraphically triggered, contrast agent-e
beam CT. Radiology 1995;196:707-14.

10 Chernoff DM, Ritchie CJ, Higgins CB. Evaluation of electron beam CT coronary angiography in healthy subjects. Am f Radiol 1997;169:93-9.

11 Nakanishi T, Ito K, Imazu M, et al. Evaluation of coronary artery stenoses using electron-beam CT and multiplanar reformation. F Comput Assist Tomogr 1997; 21:121-7.

12 Budoff MJ, Oudiz RJ, Zalace CP, et al. Intravenous three dimensional coronary angiography with contrast enhanced electron beam computed tomography. Am f Cardiol 1999; 83:840-5.

13 Reddy GP, Chernoff DM, Adams JR, et al. Coronary artery stenoses: assessment with contrast-enhanced electron-
beam CT and axial reconstructions. Radiology 1998;208 167-72.
14 Schmermund A, Rensing BJ, Sheedy PF, et al. Intravenous electron-beam computed tomographic coronary angiography for segmental analysis of coronary artery stenoses. $\mathcal{F}$ Am Coll Cardiol 1998;31:1547-54.

15 Rensing BJ, Bongaerts A, van Geuns RJ, et al. Intravenous coronary angiography by electron beam computed tomography. A clinical evaluation. Circulation 1998;98:2509-12.

16 Achenbach S, Moshage W, Ropers D, et al. Value of electron-beam computed tomography for the noninvasive detection of high-grade coronary artery stenoses and occlusions. $N$ Engl f Med 1998;339:1964-71.

17 Boyd D, Gould RG, Quinn J, et al. A proposed cardiac 3-D densitometer for easy detection and evaluation of heart disease. IEEE Trans Nucl Sci 1979;26:2724-7.

18 Gould RG. Principles of ultrafast computed tomography: historical aspects, mechanisms of action, and scanner characteristics. In: Stanford W, Rumberger JA, eds. Ultrafast computed tomography in cardiac imaging: principles and practice. Mount Kisco, NY: Futura Publishing, 1992:1-16.

19 Becker C, Schätzl $M$, Feist $H$, et al. Abschätzung der effektiven Dosis für Routineprotokolle beim konventionellen CT, Elektronenstrahl-CT und bei der Koronarangiographie. Fortschr Röntgenstr 1999;170:99-104.

20 Committee for Grading of Coronary Artery Disease. A reporting system on patients evaluated for coronary artery disease: report of the ad hoc committee for grading of coronary artery disease, council on cardiovascular surgery, American Heart Association. Circulation 1975;51(suppl): $5-40$.

21 Krucoff MW, Green CE, Satler LF, et al. Noninvasive detection of coronary artery patency using continuous STsegment monitoring. Am f Cardiol 1986;15:916-22.

22 Bossaert L, Conraads V, Pintens H. ST-segment analysis: a useful marker for reperfusion after thrombolysis with APSAC? The Belgian EMS study group. Eur Heart $f$ 1991; 12:357-62.

23 Achenbach S, Moshage W, Ropers D, et al. Comparison of vessel diameters in electron beam tomography and quantitative coronary angiography. Int f Card Imaging 1998;14:17.

24 Schmermund A, Haude M, Baumgart D, et al. Non-invasive assessment of coronary Palmaz-Schatz stents by contrast enhanced electron beam computed tomography. Eur Heart f 1996; 17:1546-53.

25 Pump H, Moehlenkamp S, Sehnert C, et al. Electron beam $\mathrm{CT}$ in the non-invasive assessment of coronary stent patency. Acad Radiol 1998;5:858-62.

\section{IMAGES IN CARDIOLOGY}

\section{Haemangioma of the interatrial septum}

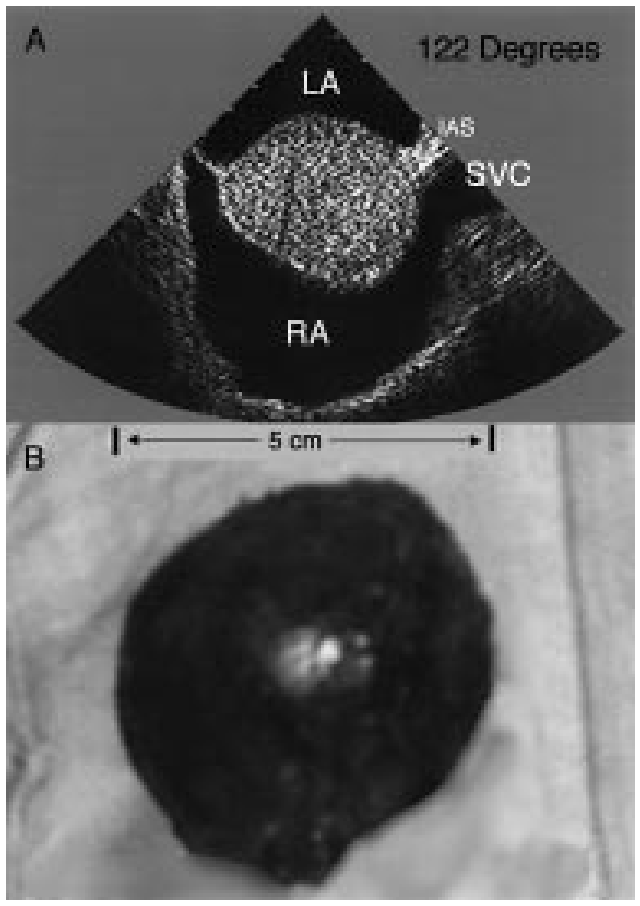

A 55 year woman was referred for evaluation of an atrial mass seen on transthoracic echocardiography. She had presented to her primary care physician with palpitations and atypical chest pain. Transoesophageal echocardiography (top) revealed a well circumscribed mass in the region of the interatrial septum (IAS). The patient was taken to the operating room. After institution of cardiopulmonary bypass, the right atrium (RA) was incised. The mass was confined to the interatrial septum. It was completely resected, and the septal defect closed with a pericardial patch. Pathologic examination was consistent with a haemangioma (bottom). The patient made an uneventful recovery and was discharged home on the fourth postoperative day. DONALD OXORN BARBARA ROBINSON GABRIEL ALDEA 